\title{
Space Weather applications with CDPP/AMDA
}

\author{
V. Génot* \\ CESR, Université de Toulouse (UPS) \& CNRS (UMR5187), 9 av. colonel Roche, BP \\ 44346, 31028 Toulouse Cedex 4, France
}

C. Jacquey, M. Bouchemit, M. Gangloff,, A. Fedorov, B. Lavraud, N. André,, L. Broussillou, C. Harvey, E. Pallier, E. Penou

CESR, Université de Toulouse (UPS) \& CNRS (UMR5187), 9 av. colonel Roche, BP 44346, 31028 Toulouse Cedex 4, France

E. Budnik

Noveltis, 2 av. de l'Europe, 31520 Ramonville Saint-Agne, France

R. Hitier

Co-Libri, 11300 Pauligne, France

B. Cecconi

LESIA, Observatoire de Paris-Meudon, 5 place Janssen, 92190 Meudon, France

F. Dériot, D. Heulet

CNES, Centre Spatial de Toulouse, 18 avenue E. Belin, 31401 Toulouse, France

J.-L. Pinçon

LPCE, Laboratoire de Physique et Chimie de l'Environnement, 45071 Orléans, France

\begin{abstract}
AMDA (Automated Multi-Dataset Analysis), a new data analysis service, recently opened at the French Plasma Physics Data Center (CDPP). AMDA is developed according to the Virtual Observatory paradigm : it is a web-based facility for on-line analyses of space physics. Data may come from its own local database as well as remote ones. This tool allows the user to perform classical manipulations such as data visualization, parameter computation and data extraction. AMDA also offers innovative functionalities such as event searches on the content of the data in either visual or automated ways, generation, use and
\end{abstract}

\footnotetext{
*Corresponding author

Email address: vincent.genot@cesr.fr (V. Génot)
} 
management of time-tables (event lists). The general functionalities of AMDA are presented in the context of Space Weather with example scientific use cases.

Key words: space physics, space weather, data analysis tool, virtual observatory

\section{Introduction}

With the growing amount of data available to researchers, scientific analyses have become a more and more complex game. The alliance between mass storage, computer power and web technologies makes however possible the design of innovative tools whose aim is to ease analysis tasks. This is also the general idea behind the Virtual Observatory paradigm (see for instance Harvey et al. (2008)).

However, while the astronomy and astrophysics domain are relatively advanced regarding their implication in the creation of Virtual Observatories (VO), space physics (and planetary physics) are just beginning their first steps into this effort which requires the adhesion of a vast community. At the French and European level, it is one of the goals of CDPP to encourage the space physics community to participate in this project. The CDPP (Centre de Données de Physique des Plasmas ${ }^{1}$ ) is the French national centre for space physics data. It was jointly created by CNES (Centre National d'Etudes Spatiales) and CNRS (Centre National de la Recherche Scientifique) in 1998; it is hosted at CESR (Centre d'Etudes Spatiales des Rayonnements, Toulouse). Recently, the CDPP has opened a new web-based service called AMDA (Automated Multi-Dataset Analysis $^{2}$; see Jacquey et al. (2009)). It is aimed to be the CDPP contribution to the Virtual Observatory in space physics.

Space Weather is a science at the interplay of several disciplinary fields : solar, solar wind, magnetospheric, ionospheric, and near-Earth environment physics. Therefore in depth studies generally request the use of data from multiple and heterogeneous origins. Such situations are typical use cases for AMDA although this tool was primarily designed for magnetospheric science (e.g. Génot et al. (2009)). In this paper we discuss how Space Weather scientists could benefit from the use of AMDA. In the next Section we briefly present the different functionalities of this tool. In Section 3 we list the Space Weather related data which can be accessed and manipulated by AMDA. In Section 4 we present a few Space Weather relevant use cases. Finally in the conclusion, and from CDPP experience in the making of AMDA, we expose the next challenges for the development of a space physics VO.

\footnotetext{
${ }^{1}$ http://cdpp.cesr.fr/

${ }^{2}$ http://cdpp-amda.cesr.fr/
} 


\section{AMDA system}

\subsection{Functionalities}

In order to enable complex operations on data, AMDA has been built around elementary objects: the parameter and the time table. A first and immediate difference relatively to most data-bases is that the concept of data files does not exist in AMDA at the user level. Indeed the user manipulates parameters (e.g. density, magnetic field, etc) without taking care of the files where they come from. Inside the AMDA system, the parameter is associated with properties (scalar, vector, tensor, units, etc) and with corresponding options (decomposition, frame of reference, etc). The second useful object in AMDA is the time table which is basically a collection of time intervals. The functionalities of AMDA allow to use and to couple these two classes of objects. They are interactively activated through web-interfaces by the user who edits requests and collects the results in his/her workspace. All the requests and results may be saved for further sessions as soon as the user has registered. We briefly describe here these functionalities. AMDA is developed in a variety of programming languages such as PHP, C, JavaScript, etc. It uses IDL for parameter computation (combination, resampling, data mining) and plot generation.

- Parameter builder. In the My Parameters interface, the user can compute new parameters by editing a mathematical expression combining existing parameters. Heterogeneous time bases are handled by AMDA in the following way : from the time start, time interval and time step chosen by the user a reference time array is built; original parameters (bricks of the new parameter) are sampled on the reference time base. If the sampling time of an original parameter is less than half the reference time step, then averaging is used, otherwise interpolation is performed. A data gap is defined by the system as equal to 5 sampling time steps of a parameter. Once a new parameter has been created it can be used as any pre-existing parameter. Usual mathematical functions are enabled but also more refined ones such as temporal shift, derivative, smooth, mean, etc. Common plasma physics constants are defined in the system and user-defined aliases may be used to simplify the editing syntax throughout AMDA (see Figure 1).

- Remote data access. Following the Virtual Observatory paradigm AMDA gives a direct access to parameters held in distant databases. In the $E x$ ternal Data interface, the user can browse through the parameters of the distant databases, select the desired ones and save them in his/her own external data tree. This data tree, with the usual hierarchy mission / instrument / dataset / parameter, will then be appended to the existing local data tree. The parameters referenced in the external data tree can be used thereafter like any pre-existing ones (see Figure 2). At present the AMDA local database may be completed by data from : $\mathrm{CDAWeb}^{3}$

\footnotetext{
${ }^{3}$ http://cdaweb.gsfc.nasa.gov/
} 
(NASA), THEMIS ${ }^{4}$ (University of Berkeley and mirror site at CESR), MAPSKP $^{5}$ (Cassini database from University of Michigan and mirror site at CESR), Venus Express magnetic field data base ${ }^{6}$ (Vex/MAG at IWF, Graz). Connections are provided by web services, ftp or NFS mounting depending on cases. Data described by the SPASE data model (Space Physics Archive Search and Extract ${ }^{7}$ ) can be easily accessed by AMDA (MAPSKP and Vex/MAG are examples). Data providers wishing to make their data attainable by/visible in AMDA should contact the AMDA team for a case by case analysis.

- Data download. In the Download Data interface, newly created, remotely accessed or local parameters can be equally downloaded (or exported) by the user in different formats (plain ASCII, CEF, CDF) and with a chosen resolution. Time series may optionally be joined on a common time basis before exporting. The data merging is performed either by averaging or interpolating, depending on the time resolution of the original datasets (see the procedure described in the 'Parameter Builder' menu).

- Data visualisation. In the Plot Data interface, the user can edit a figure combining any available parameter with the desired options (reference frame, scaling, etc). The request can be saved in such a way that the edited figure can be applied to any time interval subsequently chosen by the user. Note that the interface provides the option to apply time shifting for solar wind propagation. This functionality also accepts time tables as input. Widgets allow to skip the figure from a time interval to the next/previous one.

- Visual search. While browsing the requested figures, the user can retain intervals when a special feature, visually determined, occurs. He can do so by double clicking at the start/stop of the event. An interface is provided to store this interval in a list : the user can therefore create a time table by visual inspection.

- Automated conditional search. A second way to create a time table is by selecting time intervals when a particular condition applied on given parameters is fulfilled. In the Search in Data interface (see Figure 3), the user can edit his/her condition with mathematical functions and logical operands $(>,<$, and, or $)$. This condition is applied on a given time window and only sub-intervals fulfilling the condition are retained to populate the time table. The time window may itself be a single time interval or a time table, offering the possibility to perform successive automated

\footnotetext{
${ }^{4}$ http://themis.ssl.berkeley.edu/

${ }^{5}$ http://mapskp.cesr.fr/

${ }^{6} \mathrm{http}: / /$ www.iwf.oeaw.ac.at/en/research/solar-system/planets/venus/venus-express/vexmag.html

${ }^{7}$ http://www.spase-group.org/
} 
searches. The averaging/interpolating procedure described above in the 'Parameter Builder' menu is also used here but the definition of a data gap is let to the user (as a function of the sampling time step).

- Time table manager. In the My TimeTables interface (see Figure 4), time tables produced by visual inspection or conditional search may be edited, modified and saved. It is possible to apply operations on a single time table: extend/shrink/shift by a given duration; or on multiple time tables: union or intersection. Time tables may be imported/exported in ASCII format or in VOTable ${ }^{8}$ format (XML) compliant with IVOA standards (International Virtual Observatory Alliance ${ }^{9}$ ). Import may be from the user local disk or from an URL.

For any menu accessible in the upper frame a user help is available and will pop up in a new window.

\subsection{Access and data policies}

Using AMDA is free. However because each user is offered a workspace on CDPP disks each access needs to be authenticated. This workspace is saved from session to session and holds the production of the user : new parameters, time tables, plotting or searching requests. Therefore this requires a personal registration at AMDA service which is simply done by mailing at amda@cesr.fr. However for system evaluation purposes a 'guest login' procedure (without registration) is available with limited access time and request functionalities.

In the case of private data (PI-ownership, confidentiality, embargo) access may be restricted by the management of specific user rights.

As described below AMDA gives access to data held in remote data centers. Data use policies from these centers must then be followed by AMDA users and are clearly visible on the site. Finally AMDA team likes to be acknowledged in any scientific production (papers, presentations, web sites, etc) based on AMDA usage and made public.

\section{Space Weather-related data accessible via AMDA}

The functionalities of AMDA described above may be applied to a variety of data. These data are either stored locally (AMDA local base) or may be accessed remotely as explained in the Section above. The main remote/external database for Space Weather application is CDAWeb. We list below some parameters relevant for Space Weather studies which can be accessed by AMDA :

- geomagnetic indices (see Table 1). OMNI ${ }^{10}$ parameters (1h and $5 \mathrm{~min}$ ), and among them indices (Kp, sunspot number, etc), are also accessible;

\footnotetext{
${ }^{8}$ http://www.ivoa.net/Documents/latest/VOT.html

${ }^{9}$ http://www.ivoa.net/

${ }^{10}$ http://omniweb.gsfc.nasa.gov/
} 
Table 1: Geomagnetic indices in AMDA local base.

\begin{tabular}{cccc}
\hline Indice & Time resolution & Time span & Origin \\
\hline AE & $60 \mathrm{~s}$ & $31 / 12 / 1977-31 / 07 / 2009$ & WDC Kyoto \\
AL & $60 \mathrm{~s}$ & $31 / 12 / 1977-31 / 07 / 2009$ & WDC Kyoto \\
AU & $60 \mathrm{~s}$ & $31 / 12 / 1977-31 / 07 / 2009$ & WDC Kyoto \\
Dst & $1 \mathrm{~h}$ & $01 / 01 / 1977-30 / 09 / 2007$ & WDC Kyoto \\
Asy H & $60 \mathrm{~s}$ & $01 / 01 / 1981-31 / 01 / 2009$ & WDC Kyoto \\
Asy D & $60 \mathrm{~s}$ & $01 / 01 / 1981-31 / 01 / 2009$ & WDC Kyoto \\
Sym H & $60 \mathrm{~s}$ & $01 / 01 / 1981-31 / 01 / 2009$ & WDC Kyoto \\
Sym D & $60 \mathrm{~s}$ & $01 / 01 / 1981-31 / 01 / 2009$ & WDC Kyoto \\
PCN D & $60 \mathrm{~s}$ & $01 / 01 / 1975-19 / 11 / 2008$ & DMI \\
AM D & $3 \mathrm{~h}$ & $01 / 01 / 1975-31 / 12 / 2008$ & ISGI \\
AA D & $3 \mathrm{~h}$ & $01 / 01 / 1975-31 / 12 / 2008$ & ISGI \\
\hline
\end{tabular}

- low and high energy particle fluxes from the THEMIS mission (full database at CESR) : ESA and SST experiments with energy up to $6 \mathrm{MeV}$;

- electron and proton fluxes and magnetic field of satellites GOES 6 to 12 spanning a period from end 1992 to now (CDAWeb);

- LANL satellite geosynchronous particle data (Magnetospheric Plasma Analyzer and Synchronous Orbit Particle Analyzer, covering keV to $\mathrm{MeV}$ energies) spanning a period from 1993 to 2008 (CDAWeb);

- CRRES satellite electron fluxes in the range 0.1-2 MeV (CDAWeb);

- Space Environment Monitor (SEM) data from the NOAA satellites spanning a period from 1978 to 2005 (CDAWeb);

- and many more.

\section{Example Space Weather use cases}

In the following we show how Space Weather oriented analyses may be performed with AMDA.

\subsection{Conditional search use case 1 : Electron sensor contamination during Solar Proton Events}

Here we focus on GOES-10 5-minute averaged data : integral electron flux with energies greater than $2 \mathrm{MeV}$ and differential proton counts (0.7-4 MeV). The target database is CDAWeb. During Solar Proton Events (SPE) the energetic ions can penetrate the electron detectors leading to elevated electron flux observations. This phenomenon is called contamination and temporarily invalidates electron data. We use AMDA to discover such contamination cases.

In AMDA Conditional Search module we set the criterion 
The first parameter is the electron flux whereas the second parameter is proton counts; the names correspond to CDAWeb syntax and are simply obtained by mouse clicks in the dedicated AMDA interface (see Figure 3). Units are in $1 /\left(\mathrm{cm}^{2}\right.$.s.sr.Mev) and $1 /\left(\mathrm{cm}^{2}\right.$.s.sr $)$ for protons and electrons, respectively. The search is performed with a 20 min sampling time and for the time period covering years 2001 and 2002. This means that the data (parameters E2 and P1) of the dataset CDAWEB:GO_KO_EP8 are downloaded (ftp protocol) then resampled on a reference time base with 20 min time step for years 2001 and 2002. The intersection over this time base between both conditions above is calculated and after a few minutes we obtain the following time table :

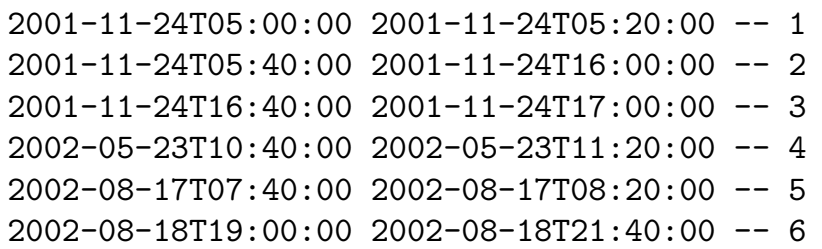

The criterion above is very rough and other events than contamination by SPE could be obtained by the automatic search. This is provided for illustration and requires refinement in the objective of a scientific study. On Figure 5 proton and electron fluxes are plotted for the 23-05-2002 event : they display similar signatures revealing the contamination of the electron sensor by energetic protons.

To confirm this automatic detection by AMDA we compare the above list to the Solar Proton Events list provided by NOAA ${ }^{11}$ : it holds 41 events for the years 2001-2002. The proton event of 24-11-2001 (events 1, 2 and 3 of the above time table) corresponds to a SPE and is known to be responsible for the contamination of electron fluxes measured onboard another spacecraft (LANL1994-084); it has been independently studied by Sicard et al. (2006). The 23-05-2002 event corresponds also to an SPE. The last two events are not listed in NOAA SPE list : they both correspond to correlated enhanced electron and proton fluxes but with smaller values than during SPE; these events need more analysis and the eventual cause of contamination should be investigated.

On a technical point of view, for such long term automatic search in data located in remote databases (in the above example, CDAWeb) the response time is highly dependent on network connections while format conversion, mathematical computation and condition testing are quick. Indeed all data for the chosen period (here 2 years) need to be downloaded before the analysis can start. Studies are underway to minimize data transfer from remote centers, for instance by keeping a proxy database of summarized parameters.

${ }^{11}$ http://www.swpc.noaa.gov/ftpdir/indices/SPE.txt 
To obtain higher quality data (sanitized from such contamination problem for instance), specialized database like IPSAT may be used. The main goal of IPSAT (Ionising Particle in SPace Analysis Tool ${ }^{12}$ ) is to allow easy access, simple analysis and plotting of in-situ space environment measurement from various sources (however IPSAT is currently not a data downloading resource).

\subsection{Conditional search use case 2: Magnetopause crossings at geosynchronous orbit}

For very disturbed solar wind conditions (high ram pressure and/or large southward component of the Interplanetary Magnetic Field) the magnetopause may be encountered at geosynchronous orbit $\left(R \simeq 6.62 R_{E}\right)$. This may have damaging consequences for spacecraft orbiting in such places as they may not be designed for solar wind / magnetosheath plasma environment. Also, such events may be associated with enhanced activities in the near Earth environment down to the lower ionosphere and, as such, are interesting in a Space Weather oriented perspective.

AMDA is tailored for event recognition such as magnetopause crossings (MPX). For this illustration we use plasma parameters from geosynchronous spacecraft to detect the crossing : for an inbound traversal the density is expected to decrease and the temperature to increase in the magnetosphere. A similar methodology using the density/temperature ratio for electrons and ions has been described elsewhere (Suvorova et al. (2005)). Here we propose a refined approach by defining the parameter $C_{\mathrm{MPX}}$ :

$$
C_{\mathrm{MPX}}=\frac{1}{T / N} \frac{\partial T / N}{\partial t}
$$

Therefore $C_{\mathrm{MPX}}$ is expected to present a positive/negative spike for inbound/outbound crossings and the typical time scale of the magnetopause crossing is given by $1 /\left|C_{\operatorname{MPX}}\right|$. From trial and error we found from an (unpublished) extensive study using Themis data that $\left|C_{\mathrm{MPX}}\right|>0.05$ is an adequate condition to efficiently select magnetopause traversals.

In the analysis below we use plasma parameters (density and temperature) from LANL1994-084 : high energy ions in the range $130 \mathrm{eV} / \mathrm{e}-45 \mathrm{keV} / \mathrm{e}$ from the Magnetospheric Plasma Analyzer (MPA) instrument with a resolution of $86 \mathrm{sec}$ (dataset L4_KO_MPA at CDAWeb). Accordingly the AMDA syntax for $C_{\mathrm{MPX}}$ to be entered in the 'Parameter Builder' interface is the following :

deriv_(\#thip/\#nhip)/(\#thip/\#nhip)

deriv_ corresponds to the IDL routine "deriv" (numerical differentiation using 3-point, Lagrangian interpolation), \#thip and \#nhip are aliases for parameters CDAWEB:L4_KO_MPA:temp_hip (1) ( $T_{\perp}$, used in lieu of $T$ ) and CDAWEB:L4_KO_MPA:dens_hip (density) respectively. The alias functionality is particularly useful for large

${ }^{12}$ http://craterre.onecert.fr/ipsat/ 
mathematical expressions using remote database parameters for which the syntax is in itself already large.

We set the analysis period to one month (May 2000) and finally obtain the following time table :

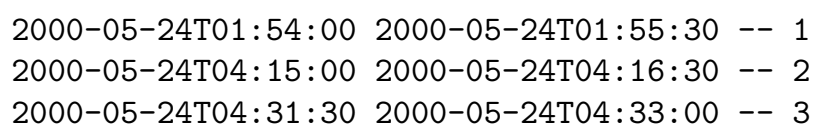

Each interval corresponds to records for which the condition $\left|C_{\mathrm{MPX}}\right|>0.05$ is satisfied. In Figure 6 we plot 4 hours of data for 24-05-2000 displaying three sharp increases/decreases in the parameter $C_{\mathrm{MPX}}$ (corresponding to the times in the above table). This event has been detected independently by Suvorova et al. (2005) using magnetic field measurements and comparing with data from a solar wind monitor (Wind). With a better resolution (magnetic field data for instance) it is possible to show that the last crossing is in fact composed of one entrance and one exit (in the magnetosphere), very close in time. Another example of multiple geosynchronous magnetopause crossings in October 2001 is displayed in Figure 7; they correspond to the time table below obtained from the same condition as above but imposed on LANL1991-080 plasma data for October 2001.

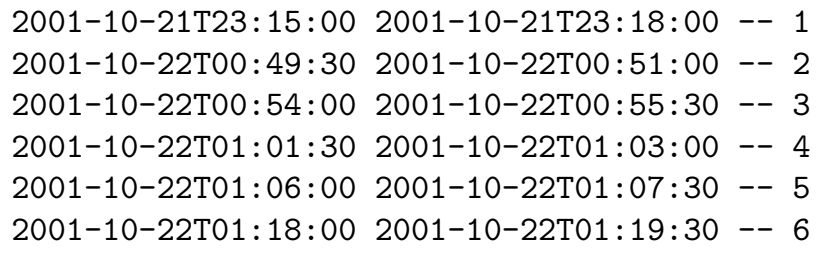

These events have also been found and analyzed in Suvorova et al. (2005).

As a consequence of this highly perturbed magnetospheric state the magnetopause crossings displayed in Figures 5 and 6 are associated with enhanced geomagnetic activity : $\mathrm{Kp}>50$ and $\mathrm{AE}>400$ for both events.

\subsection{Time table use case: Geomagnetic Sudden Storm Commencement (SSC)}

This illustration shows how to use AMDA in another way. We decided to analyse geomagnetic sudden storm commencement, a phenomenon due to pressure pulses impinging the Earth's magnetosphere. They are associated with magnetopause current increases and intensifications of the low-latitude groundbased magnetic field intensity, lasting typically for tens of minutes. The number of SSC per year is highly correlated with the solar cycle, since, as the name indicates, they are mostly observed prior to geomagnetic storms. To analyse these events we do not define an automatic recognition criterion but rather use results from the literature. Indeed, SSC lists are publicly available for instance at $\mathrm{NOAA}^{13}$. With very basic editing manipulation it is possible to transform this

${ }^{13}$ http://www.ngdc.noaa.gov/stp/SOLAR/ftpSSC.html 
list in a format directly uploadable in AMDA. Through the time table manager (My TimeTable menu) we import the following table (other time formats are allowed) :

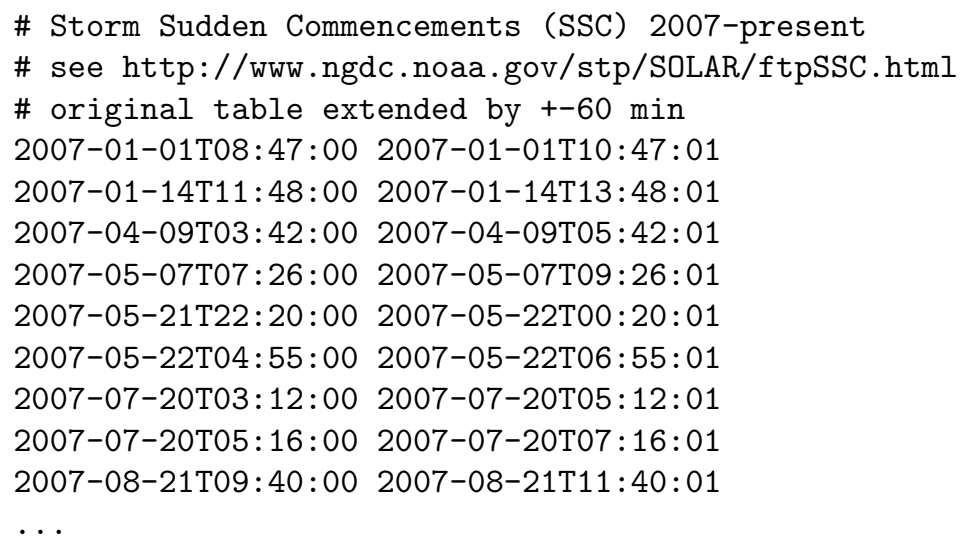

For this basic format any string (typically a text description) is accepted after the sign \#. Following a CDPP initiative VOTable (XML) is also in use. VOTable syntax is more strict but standardized which allows to communicate time tables to other VOTable compliant analysis tools such as $\mathrm{QSAS}^{14}$ and $\mathrm{CL}^{15}$ (CESR multi-mission plotting tool) and data centers such as Cluster Active Archive $\left(\mathrm{CAA}^{16}\right)$.

In the above time table we have added the context around the precise SSC time by using the Extend Intervals functionality (extending \pm 1 hour around the SSC) visible on Figure 4. From this new time table it is possible to explore different datasets to reveal consequences of SSC. For instance in Figure 8 data from GOES 11 and LANL1994-084 are plotted to show associated increase in the plasma temperature together with an intensification of $\mathrm{MeV}$ proton counts at geosynchronous orbit for the 2007-05-07 event and particular spacecraft locations. Although not detailed here, such signatures at geosynchronous orbit during SSC depend on spacecraft MLT and other parameters. Current AMDA functionalities combined with future ones, such as superposed epoch analysis, will allow to perform complete studies of signatures associated with events such as SSC.

From this time table, other functionalities of AMDA allow for instance to :

- from the Download Data menu : download any data from the local and/or remote databases for all intervals contained in the SSC time table in different common (file and time) formats. Several parameters may be aggregated in one file with a common time resolution or split in different files according to the original resolution of each parameter;

\footnotetext{
${ }^{14}$ http://www.sp.ph.ic.ac.uk/csc-web/QSAS/

${ }^{15}$ http://clweb.cesr.fr/

${ }^{16}$ http://caa.estec.esa.int/
} 
- from the Search Data menu : proceed to SSC classification by adding any further criteria on data. This will produce new time tables;

- from the My TimeTable menu : compute, for instance, the temporal intersection of the SSC table with the SEP table described in Section 4.1 to exhibit time when sudden storm commencement are associated with solar proton events; this is done by clicking both tables in the upper left menu (see Figure 4) and then activating the Intersection button; this will also produce a new time table.

\section{Conclusion}

This paper aims at introducing the basic concepts that drove the development of CDPP/AMDA and at illustrating its functionalities in the rich context of Space Weather. The ability of AMDA to connect a variety of remote databases and ingest their data in a way transparent to the user was presented. Data from heterogeneous origins may therefore be combined with complex mathematical functions to produce original parameters (which can be plotted or downloaded) or to perform searches from edited criteria. The broad spectrum of scientific instruments required to analyze Space Weather related phenomena is a perfect testbed to illustrate these functionalities.

As shown in this paper AMDA is built around two pivotal entities : the parameter and the time table. If the former is essential for scientific analyses, the latter is essential to communicate information between databases. This ability to communicate elementary bricks is the foundation on which Virtual Observatory will grow. Indeed, enhanced time table managing tools will be central in the development of the heliospheric VO, HELIO ${ }^{17}$, and in a lesser extent the planetary VO, EUROPLANET/IDIS ${ }^{18}$ (two projects in which the CDPP is involved). An important task in the building of HELIO will be the design of tools able to inter-compare time tables produced in different contexts, from the Sun to the Earth (or other planets). Such developments could pave the way for the design of a future Space Weather Virtual Observatory (such as the ESA project VISPANET).

The development perspectives of AMDA are broad. New functions (for parameter building and conditional searching) is mandatory to enlarge the panel of operations enabled by AMDA : for instance solar wind shift and a wide range of coordinate transforms will soon be available, but suggestions from the users are very welcome (mail at amda@cesr.fr). One of the next challenges of AMDA is the inclusion of, and/or connection to, models. This would be particularly interesting from a Space Weather perspective as researchers in this field are accustomed to the use of operational models (see for instance SWENET ${ }^{19}$ ).

\footnotetext{
${ }^{17}$ http://www.helio-vo.eu/

${ }^{18}$ http://www.europlanet-ri.eu/

${ }^{19}$ http://esa-spaceweather.net/swenet/
} 
Finally, the potentiality of AMDA is increased each time a new database is connected (and its parameters reachable from the External Data interface). In the Space Weather context, and more specifically regarding the radiation belt science, connection to IPSAT will be very valuable.

Finally Space Weather is no longer a science confined to the Earth environment. For instance Dubinin et al. (2009) studied the impact of solar wind corotating interaction regions on the Martian ionosphere. Also studies on Earth environment may benefit from spacecraft at Mars and Venus. For instance Futaana et al. (2008) showed how CME and flare activities on the invisible side of the Sun may affect terrestrial Space Weather. This analysis was performed with data from the ASPERA plasma instruments onboard Mars Express and Venus Express. These data are accessible in AMDA together with the magnetic field from Venus Express. Other planetary data include Cassini/MAPSKP and SKR from Cassini/RPWS. Extending the access to such data is also an important objective of the AMDA team.

\section{References}

Dubinin, E., M. Fraenz, J. Woch, F. Duru, D. Gurnett, R. Modolo, S. Barabash \& R. Lundin, Ionospheric storms on Mars: Impact of the corotating interaction region, Geophys. Res. Let., Volume 36, Issue 1, CiteID L01105, 2009.

Futaana, Y., S. Barabash, M. Yamauchi et al., Mars Express and Venus Express multi-point observations of geoeffective solar flare events in December 2006, Planet. Space Sci., Volume 56, Issue 6, 873-880, 2008.

Génot, V., E. Budnik, P. Hellinger, T. Passot, G. Belmont, P. Travnicek, P.-L. Sulem, E. Lucek, \& I. Dandouras, Mirror structures above and below the linear instability threshold : Cluster observations, fluid model and hybrid simulations, Ann. Geophys., 27, 601-615, 2009.

Harvey, C.C., M. Gangloff, T. King, C.H. Perry, D.A. Roberts \& J.R. Thieman, Virtual observatories for space and solar physics research, Earth Science Informatics, 1, 5-13, 2008.

Jacquey, C., V. Génot, E. Budnik, R. Hitier, M. Bouchemit, M. Gangloff, A. Fedorov, B. Cecconi, N. André, B. Lavraud, C. Harvey, F. Dériot, D. Heulet, E. Pallier, E. Penou \& J.-L. Pinçon, AMDA, Automated Multi-Dataset Analysis: A web-based service provided by the CDPP, in press, Proceedings of the 15th Cluster Workshop, March 2008, Tenerife, 2009.

Sicard Piet, A., S. Bourdarie, D. Boscher, \& R.H.W. Friedel, A Model for the Geostationary Electron Environment: POLE, From $30 \mathrm{keV}$ to $5.2 \mathrm{MeV}$, IEEE Transactions on Nuclear Science, vol. 53, 4, 1844-1850, 2006.

Suvorova, A., A. Dmitriev, J.-K. Chao, M. Thomsen \& Y.-H. Yang, Necessary conditions for geosynchronous magnetopause crossings, J. Geophys. Res., Volume 110, Issue A1, 2005. 


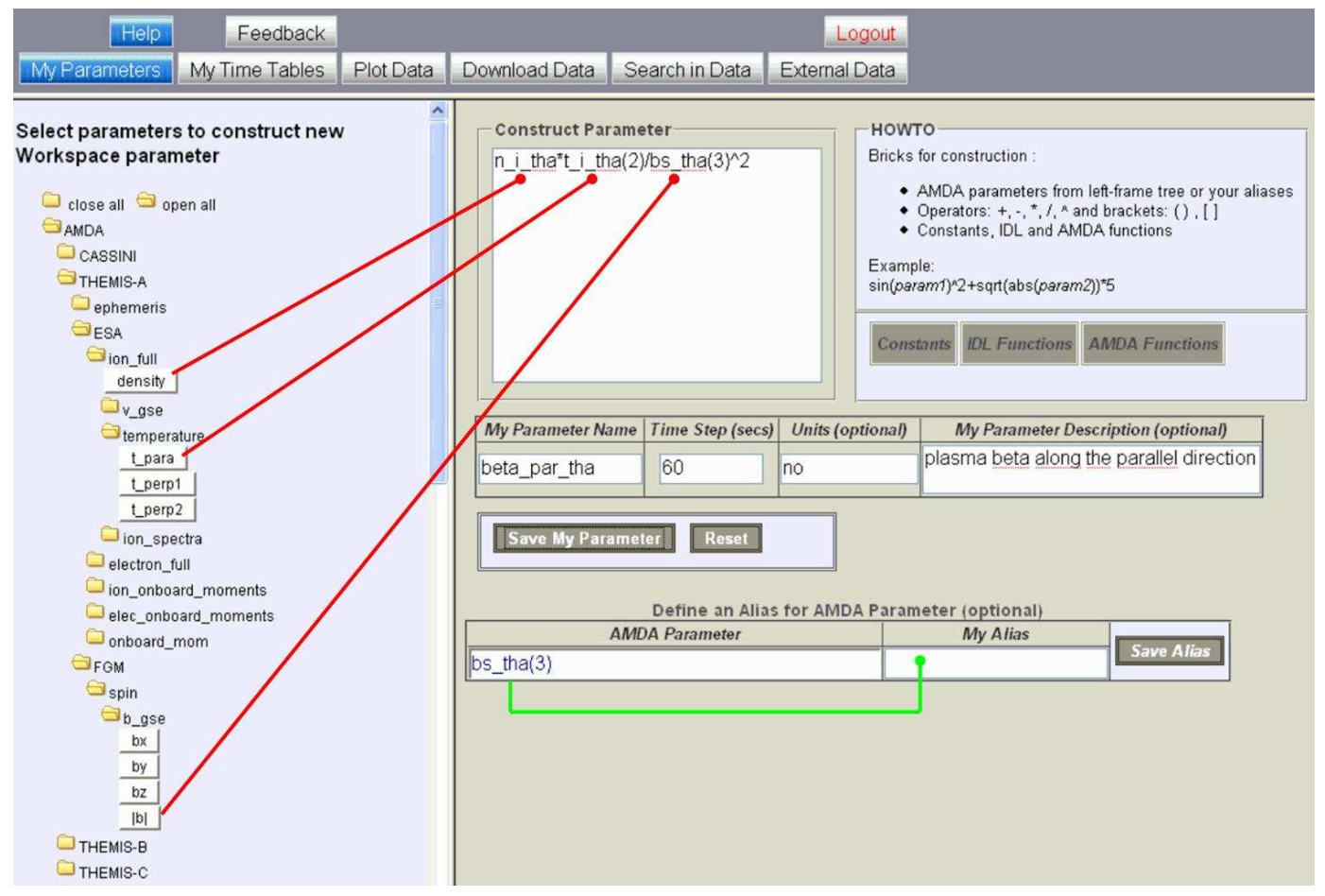

Figure 1: Parameter builder interface : the databases are displayed on the left and the hierarchical menu is expanded down to the parameter level. On the right the parameter editing window is displayed together with menus for physical constants, usual mathematical functions (IDL functions), and special functions (AMDA functions : mean, smooth, derivative, etc). Name, time resolution, unit and description are defined by the user. The bottom right menu allows to define aliases for parameters which are frequently used throughout AMDA. Once constructed the parameter is accessible in the My Parameters list (see Figure 2). The Figure illustrates how to compute the parameter plasma $\beta_{\|}$from Themis A data by clicking on density, parallel temperature (ESA instrument) and magnetic field amplitude (FGM instrument) available from the AMDA local data base. Activating the Save My Parameter button creates the new parameter beta-par-tha. 


\begin{tabular}{|c|c|c|}
\hline Help Feedback & Logout & \\
\hline \begin{tabular}{l|l} 
My Parameters My Time Tables Plot Data \\
\end{tabular} & Download Data Search in Data Exiernal Data & \\
\hline TIMAGE CDAWeb data tree & $\begin{array}{l}\hat{A} \text { My Data from External Data Centers - } \\
\text { Save Modifs }\end{array}$ & \\
\hline IINTERBAL_AURORAL & $\square$ close all $\square$ open all & \\
\hline $\begin{array}{l}\text { If INTERBALLTALL } \\
\text { IT }\end{array}$ & CDAWEB & 区 \\
\hline $\begin{array}{l}\text { If LNNL-01A } \\
\text { स LANL-02A }\end{array}$ & $\begin{array}{l}\square \text { WAND } \\
\Xi_{\text {GOES } 10}\end{array}$ & $\frac{\sqrt[x]{x}}{\sqrt{x}}$ \\
\hline (t) LANL-97A & $\mathrm{EPS}_{\mathrm{EP}}$ & 岗 \\
\hline If LANL1989_048 & $\square_{O O \_K O E E P 8}$ & 凶 \\
\hline It LANL1980_095 & OOOES_B & $\mathrm{Q}$ \\
\hline (\$) LANL1991_080 & $\checkmark$ CRRES_SATELLITE & 凶 \\
\hline •LANL1994_084 & Goes_11 & $\mathrm{Q}$ \\
\hline $\begin{array}{l}\text { E } \mathrm{SPA} \\
\exists_{\mathrm{MPA}}\end{array}$ & GLANL1994_084 & 凶 \\
\hline$\Theta_{L_{4}{ }_{1} K_{-} M P A}$ & $\Theta_{\text {MPA_KO_MPA }}$ & $\frac{x}{x}$ \\
\hline $\begin{array}{l}\text { dens_lope } \Theta \\
\text { vel_lop } \Theta\end{array}$ & $\longrightarrow$ dens_hip & 岗 \\
\hline $\begin{array}{l}\text { dens-hip } \Theta \\
\text { temen hip } \Theta\end{array}$ & $\begin{array}{l}\Xi_{\text {temp_hip }} \\
\Xi_{\text {sc_pos_geo }}\end{array}$ & $\frac{x}{x}$ \\
\hline 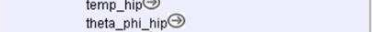 & ĐLANL1991_080__800 & $x$ \\
\hline tratio_hip $\Theta$ & THEMIS & 逐 \\
\hline $\begin{array}{l}\text { dens_e } \Theta \\
\text { tempe } \Theta \oplus\end{array}$ & GTHEMIS-A & 凶 \\
\hline theta_phi_e $\Theta$ & $\square$ FGM & $\underline{\nabla}$ \\
\hline $\begin{array}{l}\text { tratio_e } \Theta \\
\text { sc_pot } \Theta\end{array}$ & $\Xi_{\text {ions }}$ & $\frac{1 \times 1}{x}$ \\
\hline bkgd $\Leftrightarrow$ & $\beth_{\text {spectra }}$ & 凶 \\
\hline sc_pos__eo@ & $\square_{\text {electrons }}$ & 凶 \\
\hline $\begin{array}{l}\text { sc_pos_mage } \\
\text { sc_pos_syngie }\end{array}$ & $\square_{\text {ESA }}$ & $\mathrm{Q}$ \\
\hline sc_pos_syngeo $\Theta$ & ӨTHEMS-B & $\frac{x}{x}$ \\
\hline $\begin{array}{l}\text { If NOA405 } \\
\text { If NOAAD7 }\end{array}$ & $\sigma_{\text {MAPSKP }}$ & \\
\hline TOAA07 & & \\
\hline
\end{tabular}

Figure 2: Interface for the remote database parameter selector. All parameters from connected databases are visible on the left. By mouse clicking on the small arrow the user composes its own external data tree on the right (this is illustrated with the density parameter denship from LANL1994-084/MPA at CDAWeb). Once the Save Modifs button is activated the external data tree is visible throughout the different AMDA menus and its parameters can be used for plotting, building new parameters, conditional searching, and downloading. The red arrow shows how the user data tree is enlarged by clicking dens-hip. 


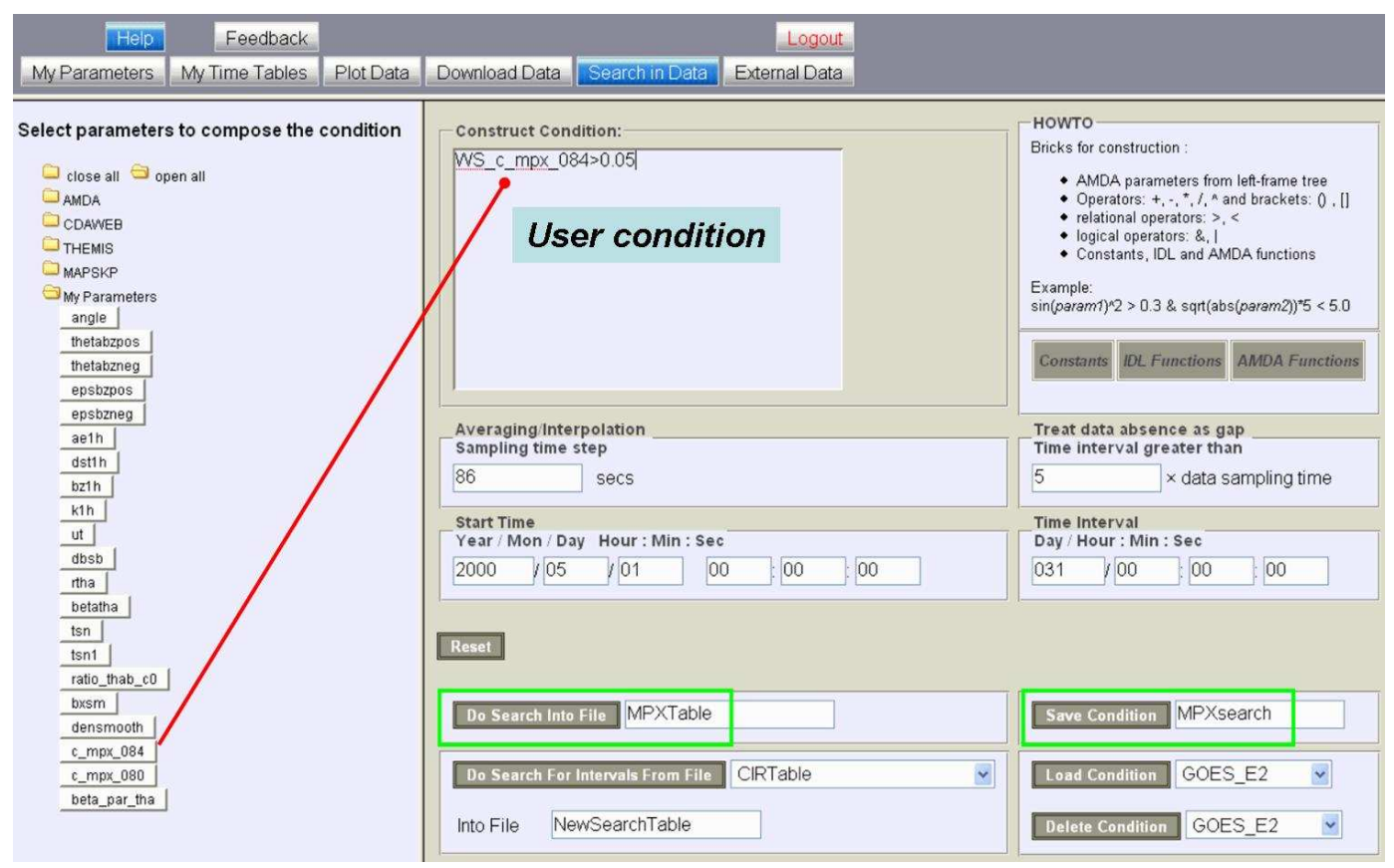

Figure 3: Conditional search interface : similarly to the Parameter builder interface the databases are accessible on the left while the condition editing window is on the right. Also the same constants and functions can be used. The sampling time step must be provided together with information on the way to treat data gaps. The condition is to be applied on a given interval or from an existing time table. The search is run when the Do Search into File button is activated. The edited condition may be saved for future use (Save Condition button). The Figure illustrates how to perform a search from a user defined parameter $\left(C_{\mathrm{MPX}}\right.$, see Section 4.2); other parameters from the database could be added to the condition. The parameter beta-par-tha constructed in Figure 1 is visible at the end of the My Parameters list. 


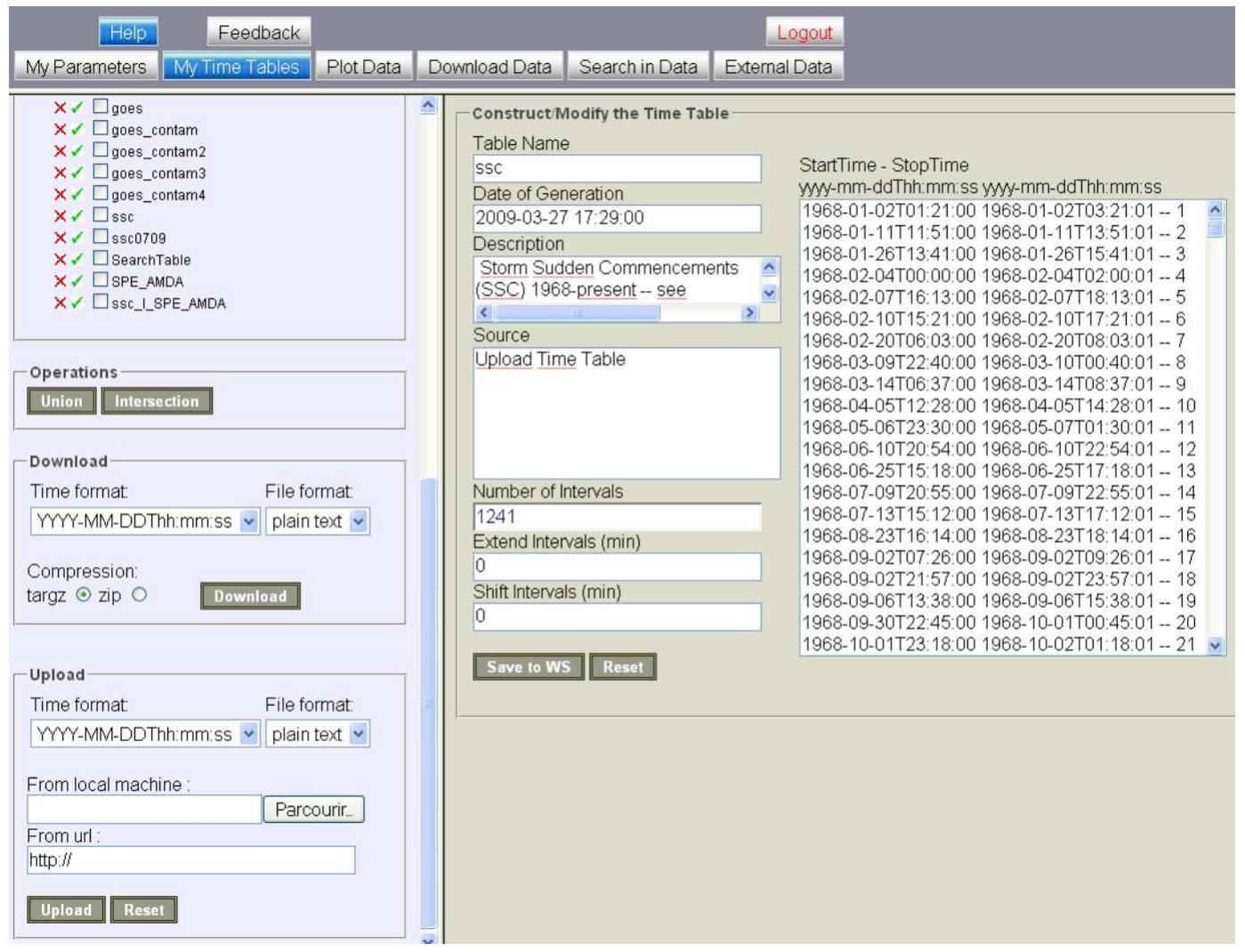

Figure 4: Interface of the time table manager. Time tables may be selected, or eventually deleted, in the upper left menu. They are edited in the right part of the window : the number of intervals and optional description are displayed in corresponding text areas. A time table may be extended or shifted by any duration and re-saved. By selecting multiple time tables on the upper left menu union and intersection operations may be applied which results in a new time table directly appended in the list. Downloading time tables is possible in various time and file formats. Uploading time table is possible from the user disk or from an URL. This example features the SSC time table described in Section 4.3. 


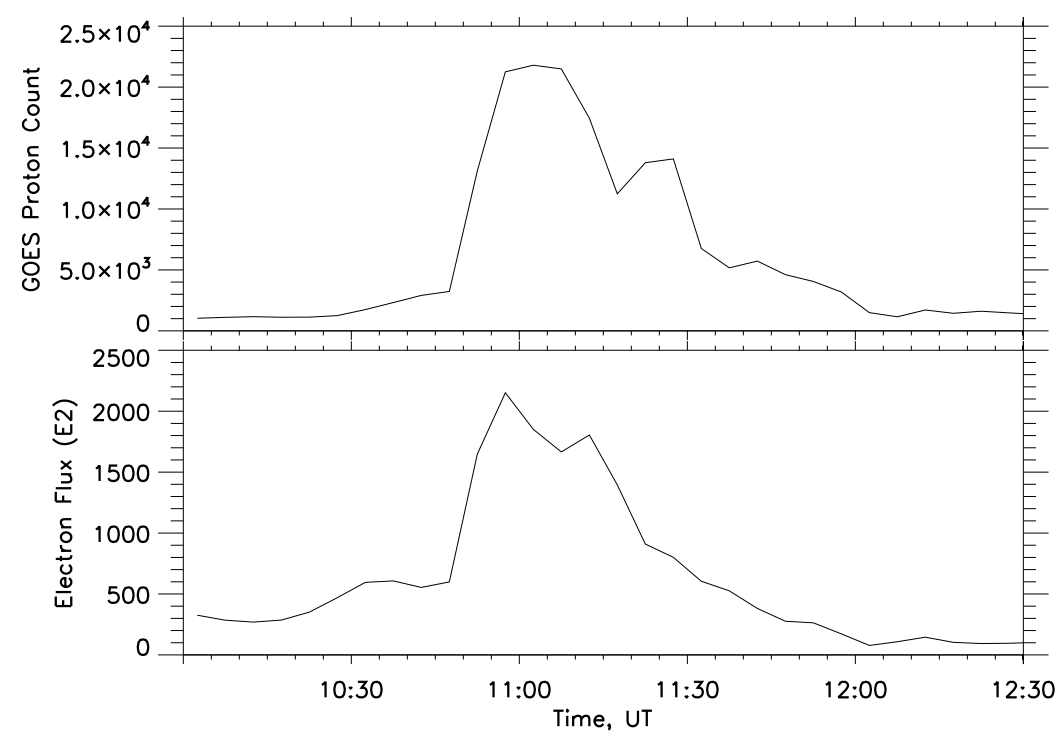

May 232002

Figure 5: Differential proton counts $(0.7-4 \mathrm{MeV})$ (top) and electron flux for particle energy $>2 \mathrm{MeV}$ (bottom) from the GOES 10/EP8 instrument for the 23-05-2002 contamination event. 


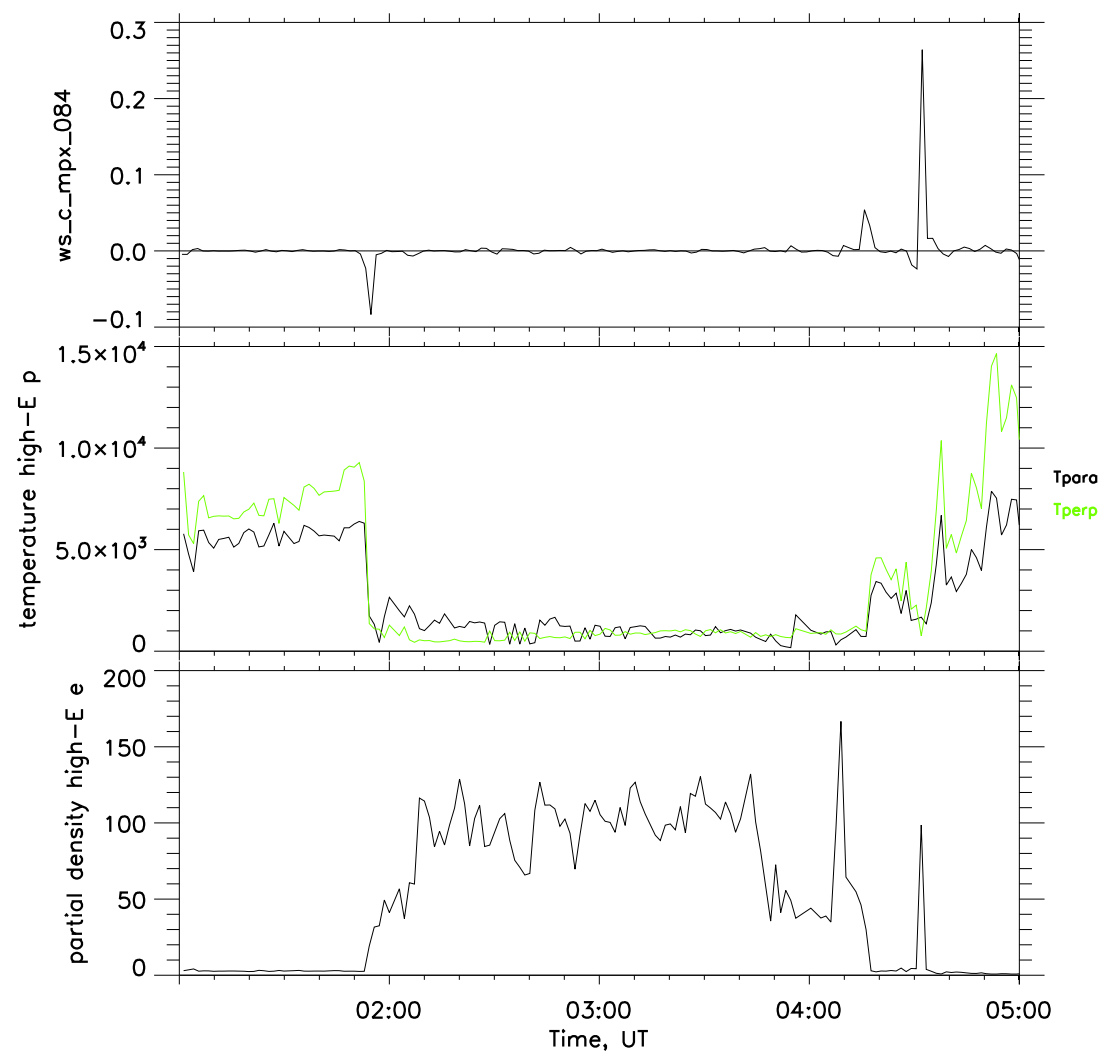

Moy 242000

Figure 6: Magnetopause crossings observed at geosynchronous orbit by LANL1994-084 on 24-05-2000. From top to bottom are plotted the value of $C_{\mathrm{MPX}}$, the ion temperature and density from the MPA instrument. Spikes in $C_{\mathrm{MPX}}$ correspond to magnetopause crossings. 


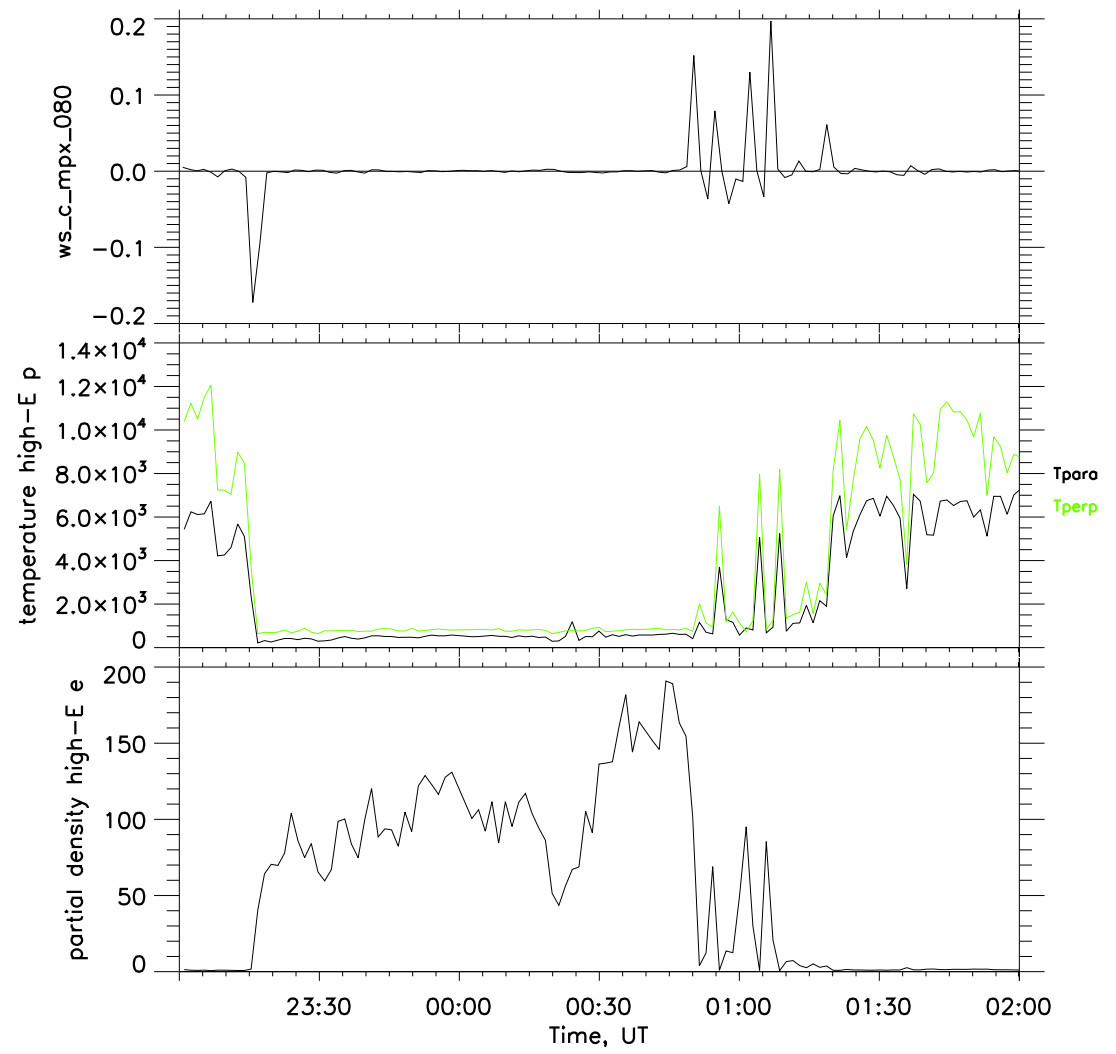

Oct 212001

Figure 7: Magnetopause crossings observed at geosynchronous orbit by LANL1991-080 in October 2001. Same parameters as in Figure 6. 


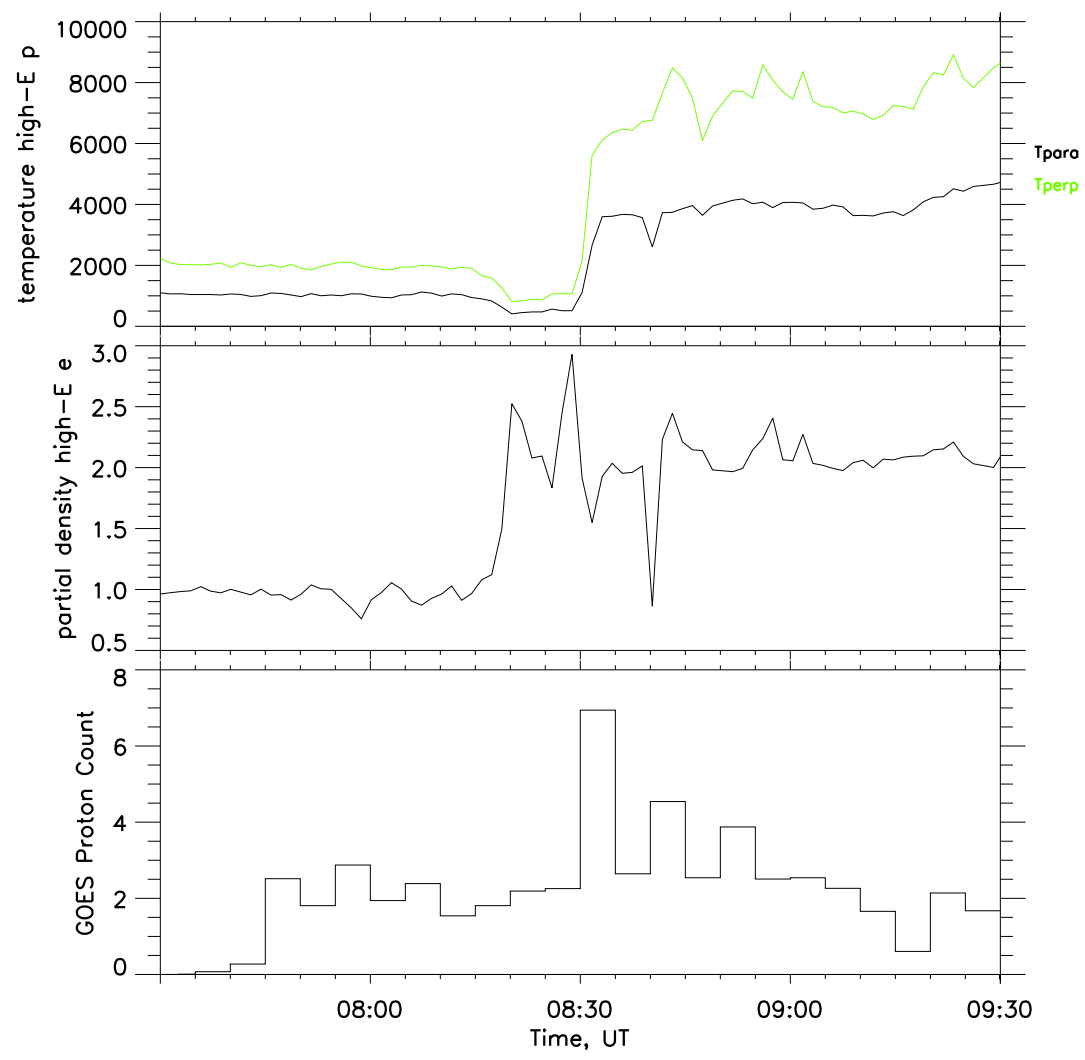

May 72007

Figure 8: Sudden Storm Commencement (at 08:26 on 2007-05-07) observed by LANL1994084 (temperature and density, top and middle panels) and GOES 11 (proton counts, bottom panel) at geosynchronous orbit. 